\title{
Erratum: Transposons: cut-and-paste gene delivery
}

\section{Caitlin Smith}

Nat. Methods 4, 183-186 (2007).

In the version of this article originally published, the statement that "At Discovery Genomics, they can create mice in which Sleeping Beauty transposons are mobilized in various tissues, which causes insertion mutations affecting endogenous genes, so that solid tumors result" was misleading. This work was done in David Largaespada’s academic lab, not at Discovery Genomics.

Also, the statement that "Sleeping Beauty transposition can create large chromosomal deletions and inversions" was incorrect. The article should have said that in some instances, Sleeping Beauty transposition can create large chromosomal deletions and inversions, though in many cases does not.

In the Suppliers guide, the listing for Clontech (BD Bioscience) should be Clontech (Takara Bio). 